\title{
A dynamic simulation approach to identify additional reactive reserves against long-term voltage instability
}

\author{
Lampros Papangelis \\ Dept. of Elec. Eng. and Comp. Science \\ University of Liège, Belgium \\ 1.papangelis@ulg.ac.be
}

\author{
Patrick Panciatici \\ Marie-Sophie Debry \\ RTE, R \& D dept. \\ Versailles, France
}

\author{
Thierry Van Cutsem \\ Fund for Scientific Research (FNRS) at \\ University of Liège, Belgium \\ t.vancutsem@ulg.ac.be
}

\begin{abstract}
A simple method is proposed to identify additional reactive reserves enabling to counteract long-term voltage instability after a large disturbance. In contrast to the many references based on power flow calculations, the method resorts to dynamic simulation. The post-disturbance system evolution is simulated in the presence of time-varying shunt susceptances with specified rate of change. This allows to deal with dynamic issues such as the required speed of the additional reactive power sources, or the onset of unstable electromechanical oscillations. Furthermore, a compromise is sought between the speed of action and the volume of additional compensation. The method is demonstrated on a detailed model of the Nordic test system.
\end{abstract}

Index Terms-long-term voltage stability, reactive power reserves, generator capability, load tap changers, time simulation

\section{INTRODUCTION}

With higher power transfers from renewable generation sites, such as large wind parks, to load centers, the existing transmission systems will be subject to power flows for which they were not designed. Hence, they will be exposed to a higher risk of instability following large disturbances. Among them, voltage instability following the loss of generation or transmission facilities, is often identified as a major threat.

Voltage stability is classified into short- and long-term [1]. The former has to do mainly with the re-acceleration of induction motors after a fault, inducing delayed voltage recovery, and in unstable cases, motor stalling. In long-term voltage instability, the initial outage and the generator reactive power limitations decrease the maximum power deliverable to loads below the level that the latter tend to restore [2].

One way of strengthening a system against voltage instability consists of increasing its reactive reserves, hosted by reactive power sources reacting automatically to disturbances [2]. While counteracting short-term voltage instability requires fast reactive power sources, such as static var compensators or static compensators, it is generally considered that long-term voltage instability can be counteracted by (the less expensive) switching of shunt compensation. There is, however, a minimal speed at which this compensation must be brought into service, in order the system to regain an equilibrium [2]. Other issues to consider are the cascade tripping of equipment by protections reacting to low voltages, or the onset of growing electromechanical oscillations.

A very large number of publications deal with the optimal location and size (and, to some extent, type) of those additional reactive power sources [3]. A majority of them are based on power flow calculations, thus neglecting the post-disturbance dynamics of the system. An optimal power flow is generally solved to find the compensation scheme that optimizes some objective [4]. The more recent references [5]-[9] have incorporated dynamic aspects, mainly in the context of short-term voltage instability.

This paper focuses on a simple method to determine where, how much and how fast additional reactive power should be injected to counteract long-term voltage instability after a major disturbance. This information is obtained by running a modified dynamic simulation of the system subject to the disturbance of concern, and supported by shunt compensation devices with a specified rate of time variation, mimicking the way compensation would be activated in post-disturbance conditions. Time simulation allows taking into account dynamic issues such as attraction towards a final equilibrium, cascade tripping of components, or the onset of unstable electromechanical oscillations.

Furthermore, although the method is not claimed to yield an "optimal" reactive compensation, a simple procedure is proposed to find a compromise between the speed of action and the volume of the additional compensation.

The rest of the paper is organized as follows. Section II presents the model of the additional reactive power sources. Section III illustrates their performance on the Nordic test system and shows the information retrieved from time simulation. A procedure to achieve a smaller compensation is presented in Section IV. Concluding remarks are offered in SectionV.

\section{MODELLING AND BEHAVIOUR OF COMPENSATION}

\section{A. Time-varying shunt compensation}

The proposed method resorts to Time-varying Shunt Susceptances (TSS) placed at various candidate locations and injecting reactive power to counteract the system degradation triggered by the initial disturbance and developing under 


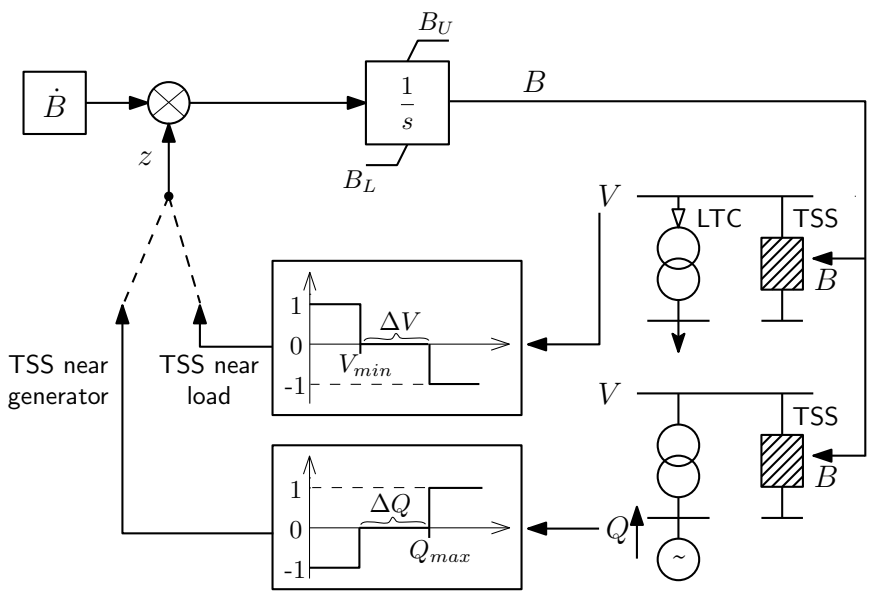

Figure 1. Model of the Time-varying Shunt Susceptance (TSS)

the effect of Load Tap Changers (LTCs) and OverExcitation Limiters (OELs) [2].

The simple model of each TSS is shown in Fig. 1. $B$ is the variable susceptance related to the injected reactive power by:

$$
Q=B V^{2}
$$

$\dot{B}$ is the specified rate of variation in time of $B$. By varying the value of $\dot{B}$, different speeds of action against the system degradation are achieved. As far as long-term voltage instability is concerned, it can be shown that slower corrective actions may have to be larger in magnitude to restore attraction towards an equilibrium [2]. Therefore, it can be expected that a value $\dot{B}$ is suitable for one contingency, but inadequate for a more severe one. Moreover, it is possible that even very high values of $\dot{B}$ cannot save the system against a contingency. Those cases usually relate to other forms of instability, of short-term nature, for which shunt compensation is not appropriate.

The variable $z$ in Fig. 1 is an integer with $z \in\{-1,0,1\}$. It takes the value 1 when reactive power must be injected into the grid, and -1 when it has to be withdrawn from the grid. Initially, the TSS is inactive with $B=0$ and $z=0$. With $z=1$, the shunt susceptance $B$ increases linearly with time at the rate $\dot{B}$, while with $z=-1$ it decreases at that rate. When the system reaches a steady-state, $z$ gets back to zero and, hence, $B$ remains constant.

The lower limit $B_{L}$ on the (non-windup) integrator is set to zero in this study, as the emphasis is on under-voltage situations where the TSS must inject reactive power. Extension to $B_{L}<0$ is straightforward. The choice of the upper limit $B_{U}$ will be discussed in Section IV. If this limit is reached, other TSS are expected to take over, but may fail doing so if the limitation is severe.

The $z$ switching logic is also depicted in Fig. 1. Two cases are considered, according to the TSS location, as detailed next.

\section{B. TSS near load}

A TSS located near a load is activated when the transmission voltage falls below a threshold value $V_{\min }$. Due to the integral control, if the system reaches a new steady state, the transmission voltage will be restored by the TSS to (at least) $V_{\min }$. The choice of this value may obey various criteria. One of them is to hold the transmission voltage high enough so that the distribution voltages can be restored at their set-points by the LTCs, i.e. the LTCs do not hit their limits and the loads are served at normal voltages. The corresponding value of $V_{\min }$ can be computed assuming the distribution transformer ratio at its limit and the distribution voltage still restored to its setpoint; a small security margin should be added.

It is possible that the combined actions of all TSS results in too high final voltages. To avoid such overcompensation, a small deadband $\Delta V$ is introduced on each TSS, as shown in Fig. 1. Thus, if the voltage rises above $V_{\min }+\Delta V, B$ is decreased at the rate $-\dot{B}$, until the voltage returns in the deadband (or the $B_{L}$ limit is hit).

\section{TSS near generator}

For a TSS of this second type, the objective is to prevent a nearby generator from getting limited, thereby losing its voltage control ability [10]. It is well known that generator reactive power limitations reduce the maximum power that can be delivered to loads, which is the root cause of longterm voltage instability [2]. Keeping generators under voltage control may be critical also for small-disturbance angle stability. It also avoids generator voltage drops that could lead to their tripping by under-voltage protections.

Thus, the activation of a TSS of this type relies on the monitoring of the reactive power $Q$ produced by the nearby assigned generator. This is shown in the lowest part of Fig. 1, where $z$ switches from zero to one when $Q$ becomes larger than $Q_{\max }$. The latter is set somewhat below the limit given by the generator capability curves. As for the other TSS, $z$ returns to zero when $Q$ gets back below $Q_{\max }$. This is where the value of $\dot{B}$ also plays a role. Indeed, either the TSS reactive injection is fast enough to prevent the limiter from acting, or the machine excitation is temporarily limited.

Furthermore, to avoid unnecessary generator relief, a small $\Delta Q$ deadband is introduced, such that, if the TSS has been previously activated (i.e. $z$ has switched from 0 to 1 ) and the generator production $Q$ falls below $Q_{\max }-\Delta Q$, the TSS will withdraw part of its reactive power injection until $Q$ returns in the deadband (or the $B_{L}$ limit is hit).

In case the generator is tripped, the TSS cannot act since there is no longer a reactive power to monitor. One option is to switch to a voltage-based activation logic, similar to that of the TSS near a load.

\section{ILLUSTRATIVE EXAMPLES OF TSS PERFORMANCE}

In this section, the operation of the TSS is demonstrated with illustrative examples using the Nordic test system, currently investigated by the IEEE PES Task Force on Test Systems for Voltage Stability and Security Analysis. The variant considered is documented in [11]. Its one-line diagram is shown in Fig. 2. It includes 74 buses and 20 synchronous machines modeled with their excitation systems, OELs, Power System Stabilizers (PSS), turbines and speed governors. The 


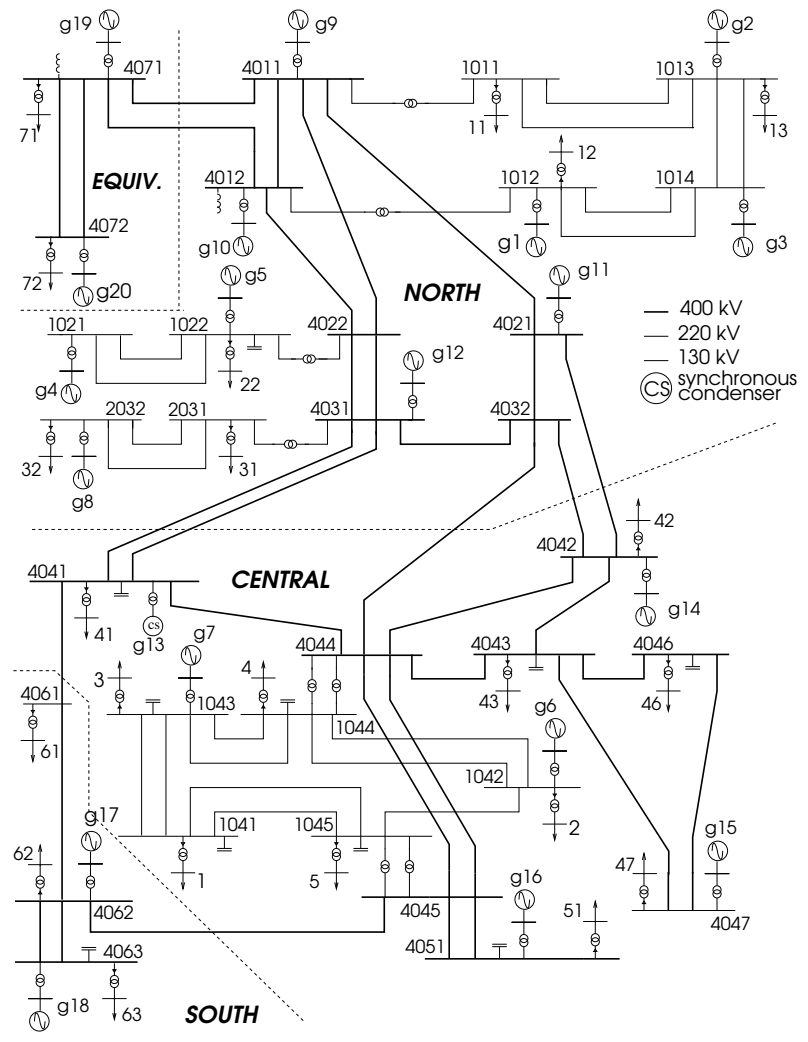

Figure 2. Nordic test system

voltage-dependent loads are represented behind distribution transformers equipped with LTCs reacting with various delays.

The insecure operating point $\mathrm{A}$, detailed in [11], has been considered, with a high power transfer from North to Central areas. A number of contingencies can lead to long-term voltage instability, mainly outages of lines in the North-Central corridor or generators in the Central or South areas. The longterm simulations were performed using the RAMSES software developed at the Univ. of Liège [12].

TSS were placed in a "brute force" manner at all transmission buses near generators and loads. However, as will be shown later, only a small number of them spontaneously react. The so reinforced system was simulated with $\dot{B}$ set to respectively $1,2,4$ and $10 \mathrm{Mvar} / \mathrm{s}$. For TSS near loads, the parameter $V_{\min }$ was set to $0.885 \mathrm{pu}$ with the objective of bringing distribution voltages back in their deadbands. In case of a generator outage, the TSS previously assigned to it injects reactive power to prevent the transmission voltage of its connection bus from dropping by more than $0.05 \mathrm{pu}$.

The first contingency considered is the tripping of line 40214042 in the North-Central corridor. Without countermeasures, it leads eventually to system collapse in less than $150 \mathrm{~s}$, as shown in Fig. 3. With $\dot{B}$ set to $1 \mathrm{Mvar} / \mathrm{s}$, the system collapse is only delayed, while setting $\dot{B}$ to $2 \mathrm{Mvar} / \mathrm{s}$ is enough to stabilize the system, even in a smooth manner, as shown in Fig. 3.

An overview of the added compensation is provided in Table I. When setting $\dot{B}$ to $2 \mathrm{Mvar} / \mathrm{s}$, it is found that only seven TSS react. They are located near generators g5, g7, g8, g10, g11, g12 and g14. For $\dot{B}=4$ and $10 \mathrm{Mvar} / \mathrm{s}$, that number decreases to five, the TSS located near g7 and g10 remaining inactive. The total reactive power added at the final, stabilized operating point, i.e. $\lim _{t \rightarrow \infty} \sum_{i} B_{i}(t)$, is reported in the table. The results confirm the already mentioned property that a faster action requires less effort than a slower one.

Table I

OVERVIEW OF TSS OPERATION IN RESPONSE TO TWO DISTURBANCES

\begin{tabular}{|c|c|c|c|c|c|c|}
\hline \multirow{2}{*}{$\begin{array}{c}\text { Reactive injection } \\
\text { (Mvar at nominal } \\
\text { voltage) }\end{array}$} & \multicolumn{3}{|c|}{$\begin{array}{c}\text { outage line } 4021-4042 \\
\dot{B}(\mathrm{Mvar} / \mathrm{s})\end{array}$} & \multicolumn{3}{|c|}{$\begin{array}{c}\text { outage gener. g17 } \\
\dot{B} \text { (Mvar/s) }\end{array}$} \\
\hline & 2 & 4 & 10 & 2 & 4 & 10 \\
\hline $\lim _{t \rightarrow \infty} \sum_{i} B_{i}(t)$ & 944 & 892 & 704 & - & 1323 & 1148 \\
\hline$\sum_{i} \max _{t} B_{i}(t)$ & 1305 & 1026 & 727 & - & 1529 & 1203 \\
\hline $\mathrm{Nb}$ of TSS reacting & 7 & 5 & 5 & - & 8 & 7 \\
\hline $\begin{array}{l}\mathrm{Nb} \text { of TSS with parti- } \\
\text { cipation at final point }\end{array}$ & 5 & 5 & 5 & - & 6 & 6 \\
\hline
\end{tabular}

The impact of the TSS speed is further illustrated in Figs. 4 and 5, showing the time evolution of the reactive power generated by g12 and the susceptance of its assigned TSS. For $\dot{B}=1 \mathrm{Mvar} / \mathrm{s}$, the system collapses before the TSS succeeds resetting the machine under voltage control. For $\dot{B}=2$ and $\dot{B}=4 \mathrm{Mvar} / \mathrm{s}$ the machine is temporarily limited, but system collapse is avoided and the machine eventually resets under voltage control. Finally, for $\dot{B}=10 \mathrm{Mvar} / \mathrm{s}$, the field current overload is alleviated before the OEL acts.

The maximum reactive power transiently injected by each TSS has been recorded, and their sum $\sum_{i} \max _{t} B_{i}(t)$ is reported in Table I. It can be seen that a fraction of the added compensation was redundant, and withdrawn before reaching steady state. This can be checked in Fig. 6 for the TSS attached to generator g14. For $\dot{B}=2$ and $\dot{B}=4 \mathrm{Mvar} / \mathrm{s}$, an overshoot of respectively 90 and 70 Mvar is observed, while for $\dot{B}=10 \mathrm{Mvar} / \mathrm{s}$, the steady-state value is reached without overshoot.

The second contingency is the outage of generator $\mathrm{g} 17$. The system is simulated for the same $\dot{B}$ values and the voltage evolution at bus 1041 is shown in Fig. 7. Setting $\dot{B}$ to 2 Mvar/s does not yield an acceptable response, since the system eventually experiences unstable electromechanical oscillations. This is attributable to a number of PSS being by-passed by the OELs, which are of the takeover type [2], [11]. By making the TSS faster, voltage control is regained, allowing the PSS to act. For $\dot{B}=4 \mathrm{Mvar} / \mathrm{s}$, six TSS are active in the final, stabilized steady state, while eight have reacted during the simulation. For $\dot{B}=10 \mathrm{Mvar} / \mathrm{s}$, those figures become seven and six, respectively. The values of $\lim _{t \rightarrow \infty} \sum_{i} B(t)$ and $\sum_{i} \max _{t} B_{i}(t)$ (see Table I) indicate that this contingency is significantly more severe.

From the extensive analysis of other disturbances, the following observations were made:

- some disturbances, such as the outage of line 40114021, result in angle instability that cannot be corrected 


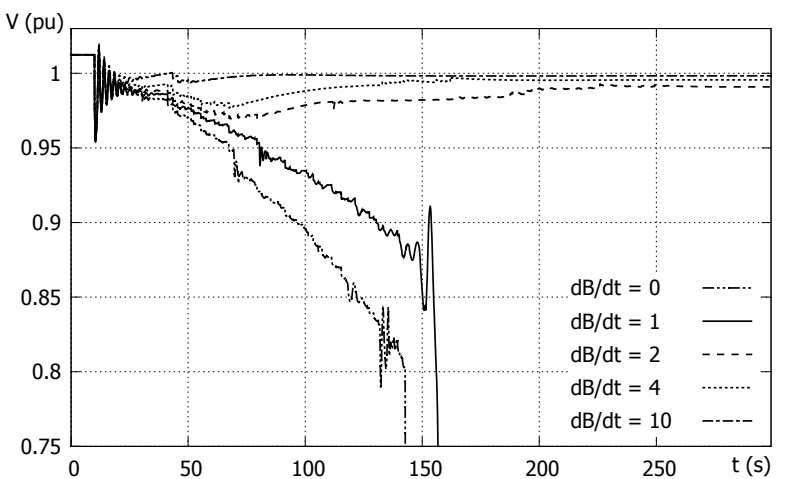

Figure 3. Tripping of line 4021-4042: voltage at bus 1041

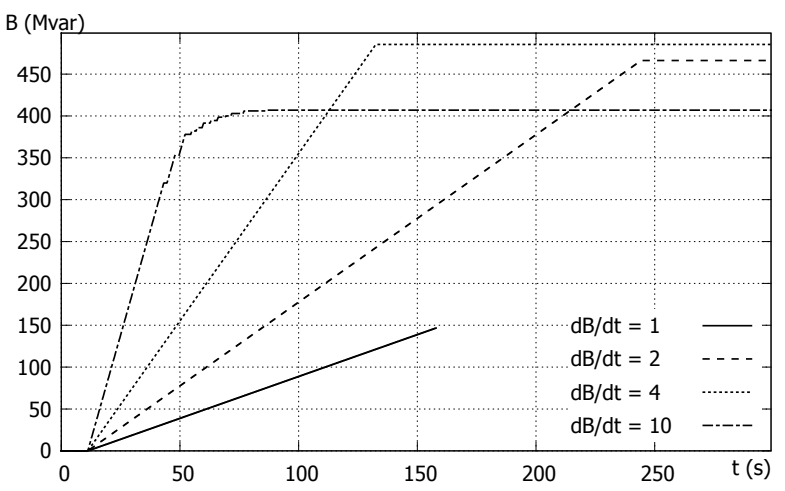

Figure 5. Tripping of line 4021-4042: susceptance of TSS next to g12

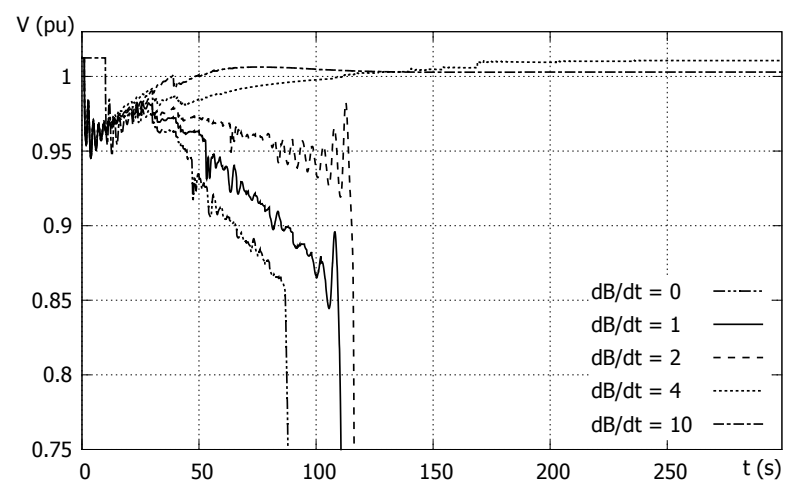

Figure 7. Outage of generator g17: voltage at bus 1041

by shunt compensation. Even high $\dot{B}$ values are unable to stabilize the system, and other emergency control schemes have to be contemplated;

- in all stabilized cases, only TSS located near generators reacted. Furthermore, if TSS were placed near loads only, some disturbances were not corrected (unless the voltage threshold $V_{\min }$ was set unacceptably high). Thus, in this system, the dominant cause of voltage instability to address is the lack of reactive reserves. This is expected to hold true in networks with "uniformly" spread generators, leading to rather short electrical distances between generation and load centers.

\section{REFINEMENT OF THE COMPENSATION SCHEME}

When designing a shunt compensation scheme, it is desirable to seek:

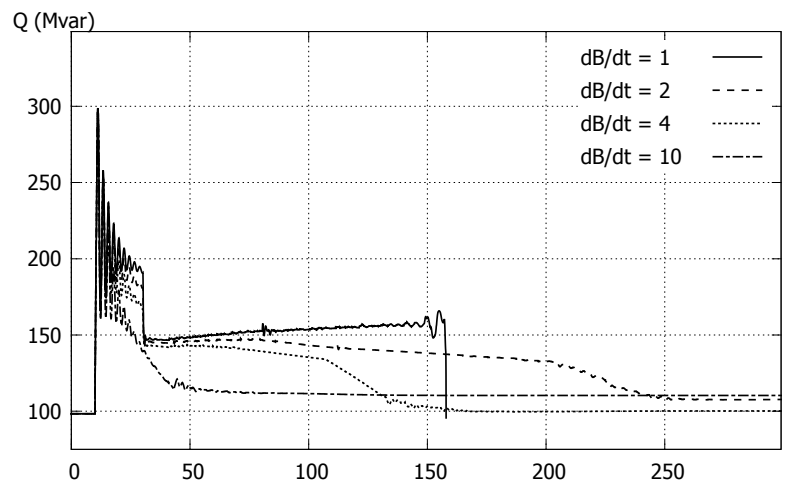

Figure 4. Tripping of line 4021-4042: reactive power of g12

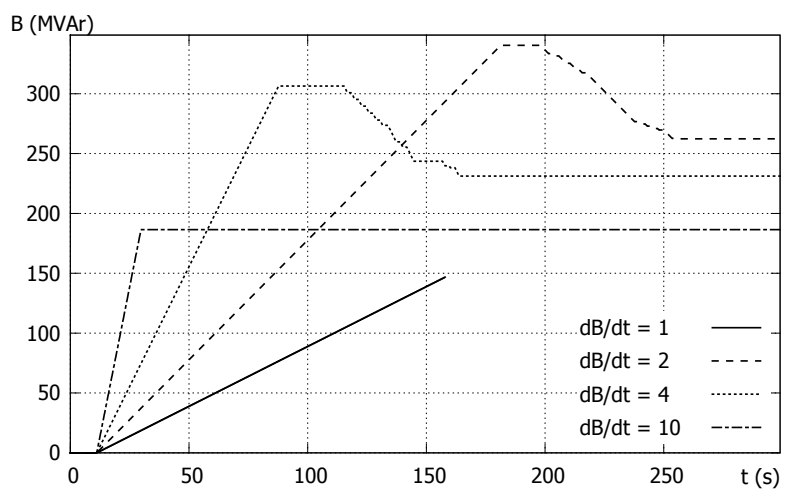

Figure 6. Tripping of line 4021-4042: susceptance of TSS next to g14

- the minimal speed of action, since a fast response may require to resort to more expensive power-electronics based devices, or may affect power quality;

- the minimal amount of reactive power, since it directly impacts the cost but also other aspects such as the space available in switching stations.

As shown previously, both objectives are conflicting. Searching for a single optimal solution would require weighting the respective costs of compensation speed and amount, which is beyond the scope of this paper. The focus here is rather on proposing a set of compensation schemes that yield acceptable system responses. To this purpose, a twophase procedure is proposed, as described hereafter. The corresponding flow charts are shown in Fig. 8.

\section{A. Phase I}

After identifying a harmful contingency, a number $N$ of TSS are placed at candidate locations near loads and/or generators. The system is simulated for various values of $\dot{B}$, without limit $B_{U}$ on the TSS (see Fig. 1) and with $B$ initialized to zero. For simplicity, the same value of $\dot{B}$ is used in all TSS. The idea is to start from a low $\dot{B}$ value and increase it by steps of $\Delta \dot{B}_{1}$ up to a maximum $\dot{B}_{\max }$. For a given $\dot{B}$ value, if the system response is acceptable, the maximum value $B_{\text {max }}=\max _{t} B(t)$ of each TSS susceptance is recorded, as well its final steady-state value $B_{s s}=\lim _{t \rightarrow \infty} B(t)$. If the response is not acceptable, $\dot{B}$ is increased and the procedure is repeated, until $\dot{B}>\dot{B}_{\max }$. The outcome of this phase is 

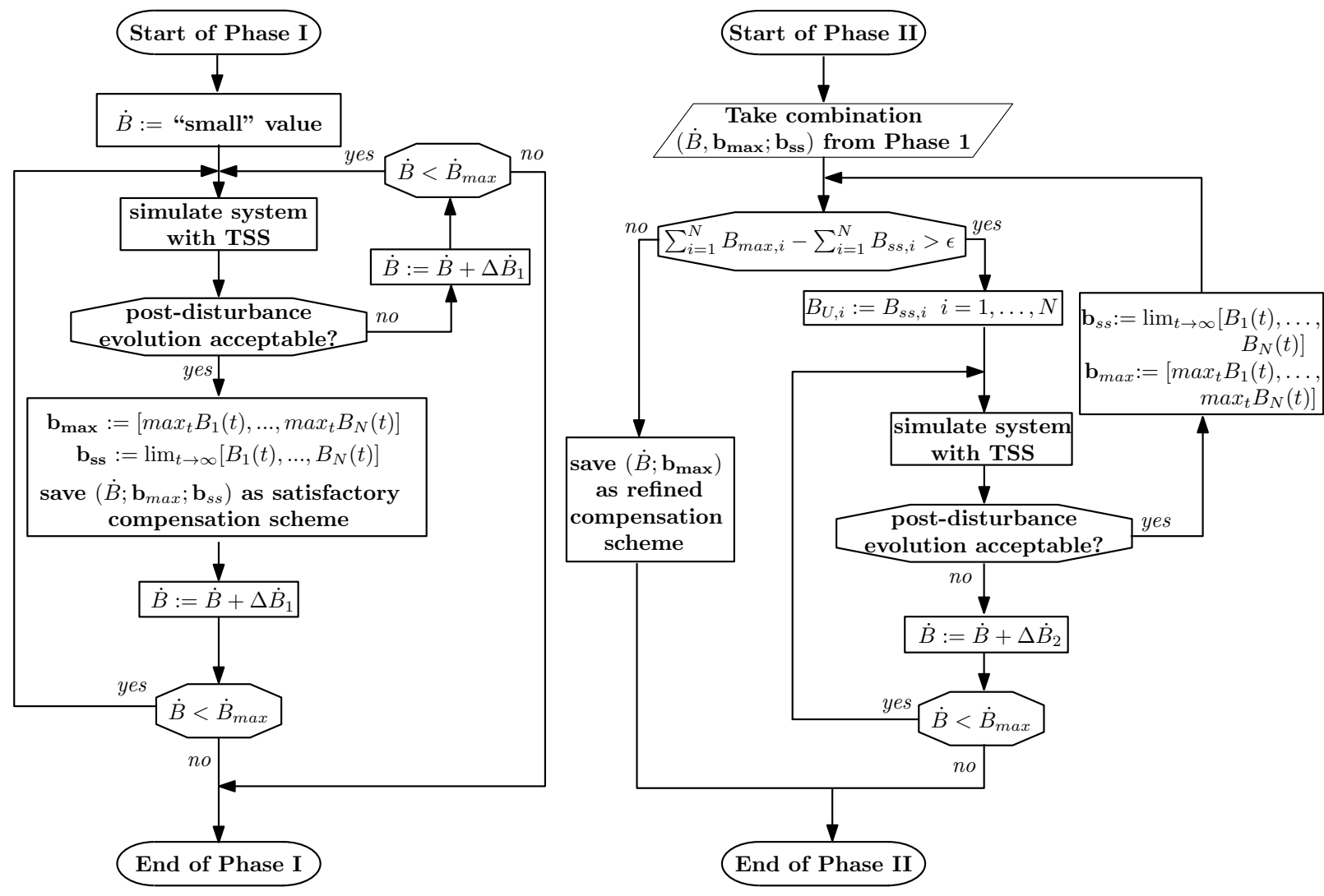

Figure 8. The two phases of the algorithm to refine the shunt compensation scheme

a set of satisfactory compensation schemes, each denoted as $\left(\dot{B}, \mathbf{b}_{\max }, \mathbf{b}_{s s}\right)$, where $\mathbf{b}_{\max }$ (resp. $\left.\mathbf{b}_{s s}\right)$ is the vector of $B_{\max }$ (resp. $B_{s s}$ ) values recorded for all TSS.

\section{B. Phase II}

In Phase I, the amount of needed compensation is set to the maximum value reached during the simulation, which is often larger than the final steady-state value. Phase II aims at determining whether this excess susceptance can be spared, while still stabilizing the system. Thus, Phase II translates each scheme $\left(\dot{B}, \mathbf{b}_{\max }, \mathbf{b}_{s s}\right)$ provided by Phase I into a scheme $\left(\dot{B}, \mathbf{b}_{\max }\right)$, in which the maximum susceptance of each TSS is fully utilized in the final steady state, i.e.:

$$
B_{s s, i}=B_{\max , i}, \quad i=1, \ldots, N
$$

It takes as input an initial compensation scheme produced by Phase I for a value of $\dot{B}$. If the total maximum compensation exceeds the total steady-state compensation by more than a tolerance $\epsilon$, i.e.

$$
\sum_{i=1}^{N} B_{\max , i}-\sum_{i=1}^{N} B_{s s, i}>\epsilon
$$

the upper limit $B_{U}$ of each TSS is set to its previously reached steady-state value $B_{s s}$, and a new simulation is performed (with $B$ initialized to zero). If an acceptable response is obtained, the aforementioned difference is checked again. If the system response becomes unacceptable under the effect of the more constraining limit $B_{U}, \dot{B}$ is increased by a step $\Delta \dot{B}_{2}$ and a new simulation is performed (unless $\dot{B}>\dot{B}_{\max }$ ). The procedure is repeated until an acceptable response is achieved. Whenever the test (3) is satisfied, the procedure stops and the currently reached compensation scheme $\left(\dot{B}, \mathbf{b}_{\max }\right)$ is the sought output. Then another compensation scheme stemming from phase I can be analyzed.

At this point, it has to be stressed that the "optimality" of the results is not guaranteed by the algorithm. Instead, it is possible that further reducing the total reactive power of the TSS could still provide an acceptable response, especially for higher values of $\dot{B}$. Moreover, the method pays more attention to generators that are operating closer to their reactive limits; the corresponding TSS will always appear in the compensation scheme, although it might not be necessary. Thus, the result of Phase II could be used as the starting point of a search aimed at further refining the compensation scheme. This possibility has not been further considered to preserve the simplicity of the method.

\section{Illustrative examples}

A sample of representative results of the proposed twophase procedure is given in Table II. They relate to three different contingencies. In Phase I, $\dot{B}$ values have been scanned up to $\dot{B}_{\max }=10 \mathrm{Mvar} / \mathrm{s}$, with a step $\Delta \dot{B}_{1}=2 \mathrm{Mvar} / \mathrm{s}$. In Phase II, a step $\Delta \dot{B}_{2}=0.5 \mathrm{Mvar} / \mathrm{s}$ has been chosen. The 
Table II

APPLICATION OF THE TWO-PHASE REFINEMENT ALGORITHM TO THREE CONTINGENCIES

$\dot{B}$ is in Mvar/s. $\quad \Sigma B_{\max }$ stands for $\sum_{i=1}^{N} B_{\max , i}$ and $\Sigma B_{s s}$ for $\sum_{i=1}^{N} B_{s s, i}$. Both are in Mvar.

\begin{tabular}{|c|c|c|c|c|c|c|c|c|c|c|c|c|c|c|c|}
\hline & \multicolumn{3}{|c|}{ Scheme 1} & \multicolumn{3}{|c|}{ Scheme 2} & \multicolumn{3}{|c|}{ Scheme 3} & \multicolumn{3}{|c|}{ Scheme 4} & \multicolumn{3}{|c|}{ Scheme 5} \\
\hline & $\dot{B}$ & $\Sigma B_{\max }$ & $\Sigma B_{s s}$ & $\dot{B}$ & $\Sigma B_{\max }$ & $\Sigma B_{s s}$ & $\dot{B}$ & $\Sigma B_{\max }$ & $\Sigma B_{s s}$ & $\dot{B}$ & $\Sigma B_{\max }$ & $\Sigma B_{s s}$ & $\dot{B}$ & $\Sigma B_{\max }$ & $\Sigma B_{s s}$ \\
\hline & \multicolumn{15}{|c|}{ Outage of line 4021-4042 } \\
\hline Phase I & 2 & 1305 & 944 & 4 & 1026 & 892 & 6 & 877 & 863 & 8 & 730 & 727 & 10 & 727 & 704 \\
\hline \multirow[t]{2}{*}{ Phase II } & 2 & 944 & 944 & 4 & 892 & 892 & 6 & 863 & 863 & 8 & 720 & 720 & 10 & 704 & 704 \\
\hline & \multicolumn{15}{|c|}{ Outage of generator g17 } \\
\hline Phase I & - & - & - & 4 & 1529 & 1323 & 6 & 1421 & 1309 & 8 & 1297 & 1214 & 10 & 1203 & 1148 \\
\hline \multirow[t]{2}{*}{ Phase II } & - & - & - & 4 & 1323 & 1323 & 6 & 1309 & 1309 & 8 & 1210 & 1210 & 10 & 1148 & 1148 \\
\hline & \multicolumn{15}{|c|}{ Outage of generator g16 } \\
\hline Phase I & - & - & - & 4 & 3185 & 2201 & 6 & 2625 & 2166 & 8 & 2365 & 2106 & 10 & 2279 & 2113 \\
\hline Phase II & - & - & - & 6 & 2168 & 2168 & 6 & 2150 & 2150 & 8 & 2106 & 2106 & 10 & 2101 & 2101 \\
\hline
\end{tabular}

tolerance $\epsilon$ has been set to 10 Mvar. The resulting sets of compensation schemes are described in Table II by the values of $\dot{B}, \sum_{i=1}^{N} B_{\max , i}$ and $\sum_{i=1}^{N} B_{s s, i}$, respectively.

Generally, it can be seen that the total reactive power needed decreases as $\dot{B}$ is increased, although the gain becomes less significant for larger values of this parameter.

It was found that, in most cases, limiting the susceptance of the TSS to the final steady-state value does not destabilize a previously stabilized system evolution. The exception was for the tripping of generator g16 with $\dot{B}=4 \mathrm{Mvar} / \mathrm{s}$. In that case, the transient overshoot of the TSS compensation was indispensable to restore attraction to an equilibrium. Phase II revealed that, after limiting the TSS susceptances, $\dot{B}$ had to be increased to $6 \mathrm{Mvar} / \mathrm{s}$ (since the values 4.5,5 and 5.5 Mvar/s also failed) in order to achieve an acceptable system response. This yields Scheme 2 in Table II. A separate run of Phase II starting from $\dot{B}=6 \mathrm{Mvar} / \mathrm{s}$ resulted in Scheme 3. However, it can be seen that the difference between both schemes is 18 Mvar, which is negligible.

\section{CONCLUSION}

A dynamic simulation approach has been presented to identify reactive reserves able to counteract long-term voltage instability after a large disturbance.

A simple technique of time-varying shunt susceptances injecting reactive power at various selected locations, in response to the system degradation has been introduced to emulate the activation of shunt compensation devices responding to degraded post-disturbance conditions. The effect of the speed at which reactive power is injected has been demonstrated through illustrative examples on a test system for voltage stability. Moreover, an iterative procedure has been described, which aims at decreasing the amount of compensation while adjusting its speed of action to guarantee the system stabilization. The outcome of this procedure is a set of satisfactory compensation schemes.

Envisaged future work will deal with:

- the possibility to arbitrate between pre- and postdisturbance injection of reactive power;
- the extension to short-term dynamics, more precisely the improvement of voltage recovery after a fault;

- the generalization of the approach to a multiple contingency framework, aiming to identifying a single compensation scheme that effectively preserves the system against a whole set of harmful contingencies.

\section{REFERENCES}

[1] P. Kundur, J. Paserba, V. Ajjarapu, G. Andersson, A. Bose, C. Canizares, N. Hatziargyriou, D. Hill, A. Stankovic, C. Taylor, T. Van Cutsem, and V. Vittal, "Definition and classification of power system stability IEEE/CIGRE joint task force on stability terms and definitions," IEEE Transactions on Power Systems, vol. 19, pp. 1387-1401, Aug 2004.

[2] T. Van Cutsem and C. Vournas, Voltage stability of electric power systems, vol. 441. Springer, 1998.

[3] W. Zhang, F. Li, and L. Tolbert, "Review of reactive power planning: Objectives, constraints, and algorithms," IEEE Transactions on Power Systems, vol. 22, pp. 2177-2186, Nov 2007.

[4] F. Capitanescu, J. M. Ramos, P. Panciatici, D. Kirschen, A. M. Marcolini, L. Platbrood, and L. Wehenkel, "State-of-the-art, challenges, and future trends in security constrained optimal power flow," Electric Power Systems Research, vol. 81, no. 8, pp. 1731 - 1741, 2011.

[5] A. Tiwari and V. Ajjarapu, "Optimal allocation of dynamic var support using mixed integer dynamic optimization," IEEE Transactions on Power Systems, vol. 26, pp. 305-314, Feb 2011.

[6] A. Meliopoulos, V. Vittal, J. McCalley, V. Ajjarapu, and I. Hiskens, "Optimal allocation of static and dynamic var resources," PSERC, Atlanta, GA, Final Project Report, 2008.

[7] S. Wildenhues, J. Rueda, and I. Erlich, "Optimal allocation and sizing of dynamic var sources using heuristic optimization," IEEE Transactions on Power Systems, vol. PP, no. 99, pp. 1-9, 2014

[8] M. Paramasivam, A. Salloum, V. Ajjarapu, V. Vittal, N. Bhatt, and S. Liu, "Dynamic optimization based reactive power planning to mitigate slow voltage recovery and short term voltage instability," in PES General Meeting - Conference Exposition, 2014 IEEE, July 2014.

[9] H. Liu, V. Krishnan, J. McCalley, and A. Chowdhury, "Optimal planning of static and dynamic reactive power resources," IET Generation, Transmission Distribution, vol. 8, no. 12, pp. 1916-1927, 2014.

[10] S. R. Islam, D. Sutanto, and K. M. Muttaqi, "Coordinated Decentralized Emergency Voltage and Reactive Power Control to Prevent Long-Term Voltage Instability in a Power System," IEEE Transactions on Power Systems, pp. 1-13, 2014.

[11] T. Van Cutsem and L. Papangelis, "Description, modeling and simulation results of a test system for voltage stability analysis," internal report, University of Liège, 2013. http://hdl.handle.net/2268/141234.

[12] P. Aristidou, D. Fabozzi, and T. Van Cutsem, "Dynamic simulation of large-scale power systems using a parallel Schur-complement-based decomposition method," IEEE Trans. on Parallel and Distributed Systems, vol. 25, no. 10, pp. 2561-2570, 2014. 